Published as: Qiang, Yi, Valcke, M., De Maeyer, P., Van de Weghe, N., 2014, Representing time intervals in a two-dimensional space: an empirical study. Journal of Visual Languages and Computing, vol. 25 (4): 466-480.

\title{
REPRESENTING TIME INTERVALS IN A TWO-DIMENSIONAL SPACE: AN EMPIRICAL STUDY
}

${ }^{1}$ Yi Qiang, ${ }^{2}$ Martin Valcke, ${ }^{3}$ Philippe De Maeyer, ${ }^{3}$ Nico Van de Weghe

1. Department of Environmental Sciences, Louisiana State University, the United States

2. Department of Educational Studies, Ghent University, Belgium

3. Department of Geography, Ghent University, Belgium 


\section{Structured Abstract}

\section{Objective}

Instead of the linear model (LM), time intervals can be represented by a two-dimensional (2D) model, which is called the Triangular Model (TM). Although the TM has been introduced for decades and applied in some areas, there still a lack of empirical studies on its usability. To fill this gap, this study aims to evaluate how people perform when using the TM to answer questions on time intervals, in comparison with using the traditional LM.

\section{Method}

Around 250 novice participants took part in the experiment, which consisted of a video training, a pretest and posttest. The video training introduced the basic knowledge of temporal relations and the two representations. The pretest allowed participants to practice the knowledge they have learned and receive feedbacks of the answers. In the posttest, participants' accuracy and speed when answering the questions were recorded for analysis. The results of using the TM and the LM were compared in pairs. The null hypothesis is that the participants produce equal results with the two models.

\section{Result}

The results showed that the participants scored better and spent less time when answering questions with the TM, which rejected the null hypothesis. Moreover, the score and speed when they used the TM did decline in the questions containing a larger number of intervals. In contrast, the score and accuracy when they used the LM declined when questions containing a larger number of intervals.

\section{Conclusion}

- The TM is easy to learn. After a 20-minute training, novice participants can use it to solve questions and produce satisfactory result.

- The TM is easy and efficient for visual queries of time intervals.

- $\quad$ The TM is easy to use for handling a large number of intervals.

\section{Implication}

- The TM can be widely applied in analysing time intervals and linear data.

- Tools implementing the TM can be learned and used by novice users. 


\section{INTRODUCTION}

A wide range of disciplines are confronted with the problem of handling information related to time, including information science (Hochheiser \& Shneiderman, 2004), archaeology (Stichelbaut \& Bourgeois, 2009) and geography (Neutens, et al., 2007). While time can be conceptualized and represented in diverse ways, the linear concept is predominant, which is reflected in many graphical representations such as time tables, chronological time lines and even the recent application in Facebook $®$ (i.e. the Facebook timeline). A segment of the time line is called a time interval, which is usually considered as the primitive of time. Up to now, considerable work has been done in handling time intervals in the areas of computer science and artificial intelligence (Bhatt, et al., 2011; De Tré, et al., 2006; Gottfried, 2008; Knauff, 1999). The most well-known work is the qualitative interval algebra introduced by Allen (1983) and the extension by Freksa (1992). Much seminal work about temporal reasoning is based on their theories. On the other hand, the research on visualization and analysis of time intervals receives far less attentions. The visual representation of time intervals remains limited to linear segments along a one-dimensional (1D) time line, which is labelled as the Linear Model (LM) in this paper. Alternative representations of time intervals are available; e.g. the cyclic representations (Li \& Kraak, 2008; Weber, et al., 2001) and calendars (Weaver, et al., 2006). They rather focus on the representation of specific aspects of time-dependent data, and are therefore not applicable in a broader range of contexts. In the LM, the second dimension is exploited solely to differentiate between the intervals of different events and thus has no metric temporal meaning. Therefore, the arrangement of linear segments can vary, depending on the sorting rules applied in the second dimension. This polymorphism prohibits the existence of a universal approach for visual analysis of time intervals. As a result, the linear time representation is most used for illustration, but rarely applied in analytical tasks of time intervals, especially exploratory data analysis, which greatly relies on data visualization.

To overcome these difficulties, a two-dimensional (2D) representation of time intervals has been considered. This representation maps a time interval to a unique point in a $2 \mathrm{D}$ space. This 2D representation of time intervals was initially proposed by Ligozat (Ligozat, 1994, 1997). According to Ligozat's approach, the vertical and horizontal axes respectively indicate the start point and end point of an interval. Later, Kulpa (Kulpa, 1997a; Kulpa, 1997b; Kulpa, 2006) proposed a 'midpoint-duration' approach, in which the horizontal axis indicates the midpoint and the vertical axis indicates the duration of an interval. He argues that this 
approach is advantageous in various applications and theoretical and has comprehensively elaborated its use in qualitative interval reasoning and interval arithmetic. Van de Weghe et al. (2007) labelled this representation the Triangular Model (TM) and applied it to an archaeological use case. More recently, Qiang et al. have investigated the use of the TM in reasoning about imperfect intervals (Qiang, et al., 2010) and interval analysis (Qiang, et al., 2012a; 2012b, 2013). Since the TM displays a set of intervals within a stable point structure, it offers special insights into interval distributions, particularly when a large amount of intervals are represented in it. Moreover, the TM supports a graphical query mechanism that relies on the manipulation of geometries in the $2 \mathrm{D}$ space. This query mechanism can help users interactively explore the dataset and exam the detected patterns. The practical use of the TM has been demonstrated in Qiang et. al (2012a; 2012b), where the TM is implemented in a GIS to support spatio-temporal analysis of interval-based geographic data. In these two papers, the TM has been applied to analyse the interval data generated from a Bluetooth tracking system and imperfect interval data in an archaeological database. Available research stressed the potential of the TM in visualising and analysing time intervals. However, there is a lack of empirical evidence to ground its understandability and usability. To fill this gap, we have initiated an empirical study to evaluate the TM, as compared with the conventional LM as a reference model. This paper summarizes the design and results of this empirical study.

The remainder of this paper is structured as follows. Section 2 introduces the basic concepts of time intervals and the two models. Section 3 first introduces the theoretical base of the learning process, and next describes the design of the test and research process. In Section 4, the results obtained from the experiment are presented and analysed. In Section 5, we discuss the findings derived from our study. Section 6 summarises the contribution of this study and proposes avenues for future research.

\section{THE TWO TIME MODELS}

\section{Linear Model}

A time interval is an extent of time, which can be the duration of an event or the lifetime of a person. In physics and computer science, a time interval is usually abstracted as a pair of real numbers $\left[I^{-}, I^{+}\right]$with $I^{-}<I^{+} . I^{-}$is the start point of $I, I^{+}$is the end point, and the difference between $I^{-}$and $I^{+}$(i.e. $I^{+}-I^{-}$) is the duration of the interval, which is denoted as $\operatorname{dur}(I)$. The LM is derived from the experience and interpretation of time linearity, and 
intuitively represents a time interval as a linear segment along the time line. The two boundary points of the segment respectively indicate the start point and the end point of the interval (Figure 1(a)). The length of the segment expresses the duration of the interval. Since time intervals may overlap and representing multiple overlapping segments in the time line may cause difficulties for visual observation, the linear segments are often arranged at different positions in the second dimension (Figure 1 (b)). The arrangement can be decided by the properties of the intervals (e.g. the start point, end point, and the duration) or the properties of entities referenced to the intervals (e.g. the categories of the events). Therefore, the structure of linear segments is variable according to the arrangement in the second dimension. Figure 2 illustrates four different arrangements of the linear segments along the second dimension. The characteristics of time intervals are expressed by the location and extent of the linear segments in the time line.

The place to insert Figure 1

The place to insert Figure 2

Two time intervals may have different relations. In 1983, Allen specified thirteen relations between two time intervals (Allen, 1983), which have been considered as the cornerstone of many theories about temporal reasoning (Bittner, 2002; Freksa, 1992; Galton, 1990; Schockaert, et al., 2008). These thirteen temporal relations are defined by the relations of the start points, and end points between the two intervals. In the LM, these relations are expressed by the topological relations between two linear segments in a 1D space. Differing from the spatial topologies, e.g. the RCC calculus by Cohn et al. (1997), the topology of time intervals also takes account of the direction of time. Therefore, there are six pairs of selfreflexive inverse relations, except the equal relation. Figure 3 illustrates the formal definitions of the temporal relations and the corresponding representations in the LM.

\section{The place to insert Figure 3}

Two relations between pairs of intervals are conceptual neighbours if they can directly transfer into one another by continuous deformation (Freksa, 1992). For example, before and meets are conceptual neighbours because extending the earlier interval towards the later interval may cause a direct transition from the before relation to meets relation. Such direct transition is not possible from the before relation to the overlap relation, because it must pass the meets relation. Based on this definition of conceptual neighbourhood, Freksa (1992) has 
mapped the thirteen temporal relations into a nested structure, called the conceptual neighbourhood structure (Figure 4). The thirteen temporal relations can be divided into three categories, i.e. open relations, semi-open relations and the closed relation. After, before, overlaps, overlapped-by, contains and during are open relations because the two intervals in these relations do not have common boundary points (i.e. the start point and the end point). Meets, met-by, starts, started-by, finishes and finished-by are semi-open relations because the two intervals in these relations have one common boundary point. The equal relation is the only closed interval as the two intervals have two common points. It is notable that all transitions from an open relation to another open relation must pass through a semi-open relation or the closed relation. Also, every pair of inverse relations is centrosymmetric about the equal relation.

\section{The place to insert Figure 4}

\section{Triangular Model}

A time interval is defined by a pair of parameters, namely, the start point and the end point. Therefore, it is possible to map a time interval to a point in a $2 \mathrm{D}$ space, using these two parameters as the coordinate. Given a time interval $I$ in the time line, two straight lines $\left(L_{1}\right.$ and $L_{2}$ ) are projected from $I^{-}$and $I^{+}$(Figure 5). The angle between $L_{1}$ and the time line, and the angle between $L_{2}$ and the time line are both $45^{\circ}$. This angle is consistent for all intervals. Therefore, the intersection point of $L_{1}$ and $L_{2}$ is completely decided by $I^{-}$and $I^{+}$. In other words, the time interval $I$ can be represented by this point in the $2 \mathrm{D}$ space. This representation of time intervals is called the Triangular Model (TM). Because $\alpha_{1}=\alpha_{2}$, it is straightforward to deduce that the horizontal location of the point indicates the middle point of the interval, i.e. $\operatorname{mid}(I)$. In the vertical dimension, the height $(h)$ of the point is half of the length of the linear interval $(l)$, i.e. $h=0.5 \cdot l$. Thus, the height of an interval point in TM indicates the duration of the interval. Using this approach, every time interval can be represented as a unique point in the 2D space, and the characteristics of a time interval are completely expressed by the location of the point. Note that $\alpha$ can be different values for specific purposes. In this paper, we set $\alpha=45^{\circ}$, to be consistent with earlier work (Kulpa, 1997; Qiang, et al., 2010; Van de Weghe, et al., 2007). Considering this setting, the eight directions in the 2D space correspond to eight changing directions of interval properties (Figure 6). Note that, besides the 'midpoint-duration' projection of the TM, an interval can be 
projected to a 2D point by any two of the four properties, including start point, end point, duration and midpoint. Ligozat's $(1994,1997)$ 'start point - end point' projection is one of these variants. In this study, we follow the 'midpoint - duration' approach due to some of its advantages, which has been elaborated by Kulpa (1997a, 2006). The empirical study and comparison of the other approaches will be scheduled in the future work.

\section{The place to insert Figure 5}

\section{The place to insert Figure 6}

Since the TM represents time intervals as points in a $2 \mathrm{D}$ coordinate space, the relations between time intervals are expressed by the spatial relations. Given a study interval $I[0,100]$, all examined intervals are located within the isosceles triangle formed by $I^{-}, I^{+}$and the interval point of $I$. Let us consider a reference interval $I_{1}[33,66]$ and several intervals $\left(I_{2}, I_{3}\right.$, $I_{4}$ ) that are before interval $I_{1}$ (Figure 7 (a)). In the TM, $I_{2}, I_{3}, I_{4}$ are located in the zone in the left corner of the study interval (Figure 7 (b)). Therefore, it is easy to deduce that this zone (i.e. the black zone in Figure 7 (c)) encloses all intervals that are before $I_{1}$. In like manner, all Allen relations with respect to an interval can be represented by such zones in the TM (Kulpa 1997a, 2006) (see Figure 8). In each diagram in Figure 8, the reference interval $I_{1}$ has been chosen in the centre of the study period to avoid visual bias. Each black zone represents the set of intervals that are in a specific relation to $I_{1}$. These zones are called relational zones. The boundaries of the relational zones are in $45^{\circ}$ or $45^{\circ}$ angle to the horizontal axis. It is noteworthy that the zones of the open relations are 2D geometries (i.e. triangle or rectangle), whilst the zones of the semi-open relations are 1D geometries (i.e. line). The zone of the closed relation (i.e. the equal relation) is a point (0D). Moreover, the zones of a pair of inverse relations are centro-symmetric about the reference interval. In Figure 8, the reference interval $I_{1}$ is chosen in the centre of the study area in order to avoid visual bias. Of course the reference interval can be put in other locations. However, the topological configuration of its relational zones remains the same (Figure 9). This way, the TM transfers a temporal topology to a spatial topology between points and zones. The intervals in a specific relation to the referenced interval can be found in a specific zone.

The place to insert Figure 7

The place to insert Figure 8 


\section{EMPIRICAL STUDY}

The potential and advantages of the TM in view of the analysis of time intervals has been elaborated in previous literatures (Qiang, et al., 2010; 2012a; 2012b). Following the TM, every interval is represented by a point at a specific location. Therefore, a set of time intervals is represented as a stable structure of points, facilitating the visual observation and analysis of interval distributions. This feature is particularly useful when comparing two sets of intervals. The pattern differences can be easily observed from the structural difference of the two point sets, which is not possible within the LM where the linear segments do not have a fixed location. Also, the TM transfers temporal topology to spatial topology between points and zones. The intervals that are in a specific relation to a reference interval can be found in a specific zone, which allows one to visually query the interval visualization. In addition, the points in the TM are apparently more space-efficient than linear segments, making it capable of analytical tasks involving large datasets of intervals.

Though these merits have been demonstrated in previous studies, there is a lack of empirical evidence that the TM can be easily processed and manipulated by non-experts. This evidence may support the feasibility of implementing the TM in analysis tools and introducing the tools to a broader range of users. To the best of our knowledge, till present, no empirical work has been done to evaluate any $2 \mathrm{D}$ representations of time intervals similar to the TM. To this end, we have conducted an empirical study that aims to assess the understandability and usability of the TM, as compared with the conventional LM. More specifically, this empirical study aims to address the following questions: 1) whether people easily learn the $\mathrm{TM}$; 2) whether people are able to correctly use it to answer question about time intervals; 3 ) whether the TM is more efficient for answering these questions; 4) whether people like to use it.

\section{Hypotheses}

Representations are central to the development and storage of conceptual knowledge. Representations help to process new information in working memory. More in particular, representations support the processing of complex information since they directly support the visuo-spatial sketchpad in working memory. In addition, they are easily stored in and retrieved from long-term memory (Baddeley \& Hitch, 1974; Miyake \& Shah, 1999). As such, 
complex knowledge, such as processing time intervals, will be fostered when learners are presented with efficient representations. The quality of representations determines the efficiency in cognitive processing. Following cognitive load theory, presenting learners with well-developed visual representations especially helps to reduce extraneous cognitive load that hinders the active processing and consecutive storage and retrieval of complex knowledge (Sweller, 1994; Sweller \& Chandler, 1994). Lastly, the Cognitive Theory of Multimedia Learning (CTML) stresses the critical need to present learners with clear multimedia (e.g. visual representations) to develop the organization of complex information into mental models that can be linked to prior knowledge in long-term memory (Mayer \& Moreno, 2003). CTML explicitly puts forward the potential of multimedia representations (such as a graphical representation of time intervals) to foster knowledge processing, resulting in better knowledge retrieval and processing.

Putting the above theoretical frameworks in the context of learning about time representations, the question can be put forward to what extent there is a differential impact of learning with the support of the LM or the TM. The LM representation is already available in long-term memory and can easily be accessed from working memory. The fact learners are acquainted with the LM, suggests that they will solve efficiently and effectively time interval problems. In contrast, the TM might invoke extraneous cognitive load due to the unknown nature of the representation. But, since no empirical research is available as to the efficiency and efficacy in processing the TM, the actual situation greatly depends on the comprehension and usability of this alternative time representation. If the TM succeeds in representing in a better way time intervals, it can be suggested that this multimedia representation is an even more efficient and effective representation and additionally takes away extraneous cognitive load. The former implies that two competing hypotheses can be put forward. As such, the presented study aligns with the need to compare the efficacy and efficiency of both representations in solving interval problems. Consequently, this study aims to test the following null hypotheses: (1) participants produce equal accuracy of results when using the two models to solve interval questions; (2) participants spend equal time using the two models to solve interval questions; (3) participants do not have a special preference to either model; (4) time use of participants equally correlates to the volume of information represented in the two models. Besides, according to the available theoretical base, a learning phase needs to be conducted prior to the comparison of the LM versus the TM. 


\section{Research Material}

A pretest-posttest intervention study was set up. The pretest and posttest aimed at measuring the comprehension and usage of the LM and the TM within a group of participants. In the tests, time intervals were represented in either of the two models. Participants were requested to answer specific questions with respect to these time intervals. Every question was asked twice with the same number of intervals presented, but shifting the use of the LM and the TM. When comparing participant comprehension and usage, the following corresponding parameters could be compared: correctness of the answer, time the participant spent on the question and the preference for a model by the participant. Parameters obtained from the two models were compared using appropriate statistical tests, building on the null hypothesis stating that the parameters from the two models are not significantly different.

Two categories of questions were included in the tests. The first type aimed to evaluate how well the participants could identify the properties of time intervals in the two models, i.e. the start point, the end point, the duration and the midpoint, which are considered as the elementary properties of time intervals in previous research about temporal reasoning (Freksa, 1992). In this type of questions, given a number of time intervals, the participants were requested to find intervals with the specific properties. The second category aimed at evaluating how well the participants could identify the temporal relation between time intervals with the two models. Participants were requested to find the intervals that satisfied certain relations to the reference intervals. Each question category consisted of two sub-types, which either asked about one item (i.e. property or relation) or two. Table 1 exemplifies the questions types. The asked items are highlighted with bold font in the example questions, which can be replaced by other items in other questions. In order to reduce the total number of questions, the questions including two temporal relations only include open relations introduced in Section 2.1.

\section{The place to insert Table 1}

Tests were presented in two modes at the time of the pretest and the posttest (Table 2). The pretest was presented as a paper-and-pencil test, containing a small number of questions with fewer intervals being presented in the two models. In the pretest, the participants were requested to answer the questions by circling the correct intervals. The posttest was presented via a website containing a set of questions (in total 37 questions) with a larger number of intervals. Due to the time limitation, every participant only needed to finish a random set of 
12 questions out of the 37 questions. The 12 questions covered all types of questions illustrated in Table 1. The composition of the 12 questions is: random 2 from Question 1-4 in Table 5, 3 from Question 5 - 10, 3 from Question 11 - 22, and 4 from Question 23 to 37.

The place to insert Table 2

In the computer-based posttest, participants were able to select the correct intervals by clicking or dragging a selection box in the diagram. When the time intervals were displayed in the LM, the participants could sort the linear segments along the vertical dimension according to a certain interval property, including the start point, the end point, the midpoint and the duration. Every question was asked twice in both the LM and the TM in a random order with the same number of intervals presented. In order to exclude extra interference, both the LM and the TM were presented in their most basic forms. Equal-interval ticks and labels were placed on the axes to indicate the scales of the axes. Gridlines from these ticks were drawn on the background in order to help the participants to read the metrics in the space. Figure 10 - 13 illustrate two example questions with intervals presented in the TM and the LM respectively. Since the study involved Dutch-speaking participants, all information and tests were presented in Dutch. At the end of the question, participants were requested to evaluate the two models by selecting one from the five options: 1): The TM is much easier; 2): The TM is a little easier; 3) They are the same; 4): The LM is a little easier; 5): The LM is much easier. The website automatically recorded participant responses, answer scores, preferences of a model and the time participants spent on each question.

The place to insert Figure 10

The place to insert Figure 11

The place to insert Figure 12

The place to insert Figure 13

\section{Participants, Instruments and Procedure}

258 participants have finished the experiment. These participants were first-year undergraduate students from Ghent University in the subject of educational sciences. Their age was close to 18 years. Although no background survey is administrated, it is reasonable to assume that they were more familiar with the LM than the TM. The reason of this assumption is that the TM is a relatively new and less-frequently used representation, and on 
the other hand, the LM and its variants have been widely and traditionally used in time representation.

The experiment starts with the training phase, which was considered to be crucial in two ways. First, it guaranteed the activation of the prior knowledge of the participants about the LM; and second, it guaranteed that a basic introduction to the TM could be implemented. The study took place in a computer lab in which every participant was allocated a desktop computer. Due to the capacity of the computer lab, the 258 students were divided into seven groups. These groups participated the same experiment at different times during a week. Students could select their participation time via an online calendar tool. To ensure every group experienced exactly the same training process, the instructions were based on standardized video clips. All students watched these video-based instructions on a central screen in front of the computer classroom. Two video-based instructions were presented to the students. In the first video instruction, students were introduced to the conceptual base about time intervals and the representations of time intervals following the LM and the TM. In the second video instruction, students were introduced to the temporal relations between time intervals and the corresponding representations following the two models. Both videos presented equal amount of knowledge about the two models, following a consistent presentation structure and format. After each video instruction, the participants were requested to answer the corresponding part of the pretest, in which the questions were related to the knowledge taught in the video clip. The results from the pretest provided a preliminary evaluation about how well the students comprehended the two models. After students finished either pretest, a feedback video was played to demonstrate the correct answers of the questions and explain the underlying rationale. This feedback video was also considered as part of the instructional intervention since it offered further chances to learn or to correct their understanding about the two models. After studying the video instructions, solving the pretests, and studying the feedback videos, students watched the instructional video of the posttest interface and then entered to the website of the posttest. The entire research procedure and time of every phase is illustrated in Figure 14.

The place to insert Figure 14

\section{RESULTS}


In this section, the result obtained from the test is presented and analysed. Since every question has been answered by participants in both the TM and the LM, it is feasible to compare the parameters obtained in these two models, including the answer score, the time used to solve the question, and the preference for a particular model. These parameters can be considered as the dependent variables in this study. The independent variables include the the shifting usage of the two models, the different questions, and the varying number of presented intervals. The comparison between the two models has been made at two levels: the entire test and individual questions. A t-test for paired samples is used to compare the scores obtained and time spent in the LM and TM. Wilcoxon signed-rank test is used to determine the preference for a particular model. The Pearson correlation coefficient is applied to indicate the correlation between the time consumption and the number of presented intervals.

\section{Test Score}

The correctness of the answer is described by a score ranging from zero to one. In the pretest, the participants produced significantly higher average scores using the LM than the TM (Table 3). However, the situations reversed in the posttest, in which the participants produced significantly higher average score using the TM (Table 3).

In the posttest, the participants produced significantly higher scores with the LM for 3 questions, while they produced significantly higher scores with the TM for 17 questions. The participants produced higher scores with the LM in the questions about the start points and end points (Question 1 and 2 in Table 4). However, in the questions about the duration and midpoint, the participants (Question 3 and 4) produced higher score when using the TM. No significant differences have been detected in most of the questions concerning two interval properties, except the questions that ask about both the duration and midpoint (Question 10) in which the TM generated higher scores. Moreover, with the TM, participants produced higher scores in 7 questions with one temporal relation (Question 11 to 22), including the relation before, meets, overlaps, started-by, during, contains, after. Only in the question about the met-by relation, participants produced higher scores within the LM. In Figure 15, we depict the mean scores of these questions as gradual colours in the conceptual neighbourhood structure of temporal relations. As can be derived from Figure 15, the mean scores obtained from the LM shows a symmetric distribution about the central point, i.e. the equal relation, which means that the participants produced similar scores in pairs of inverse relations. However, this centrosymmetry is less obvious in the TM. For instance, the 
participants produced much higher scores in the meets and overlaps relations than their inversions met-by and overlapped-by. Furthermore, in terms of the questions with two temporal relations, the scores obtained in the TM are higher than those obtained in the LM in 7 questions (Table 4). However, the opposite situation (i.e. the score obtained in LM is higher) has not been detected in any such questions.

\author{
The place to insert Table 3 \\ The place to insert Table 4 \\ The place to insert Figure 15
}

\title{
Time and Preference
}

As stated earlier, in addition to the correctness of the question answers, the time participants spent and the preference of participants for a specific model were recorded in the posttest as well. With respect to the entire posttest, the participants generally spent significantly less time when the intervals were represented in the TM (Table 3). According to specific questions, the advantage of the TM in time consumption was also overwhelming (Table 4), except the two questions that asked about the start point or end point (Question 1 and 2 in Table 4) where no significant difference was detected. On the other hand, the correlation between the number of intervals and the time used has been detected significant in more than half of the questions (20 out of 37) when the interval is represented in the LM (Table 5). Namely, the more intervals are represented in the LM, the more time the participant spent on answering the question. In contrast, such correlation has only been detected in 6 questions in the TM (Table 5), which reflects that the efficiency of the TM is less influenced by the increasing number of intervals.

In terms of the preference of the model, the participants considered the TM as the easier representation mode with respect to the entire test (Table 3). At the question level, the preference of the TM is also predominant, with the exception of 7 questions in which the participants gave a neutral opinion (Table 4).

The place to insert Table 5

\section{DISCUSSION}


In terms of the overall test scores, the reversed results have been observed in the pretest and the posttest. In the pretest, the participants produced higher scores using the LM than the TM, which could be explained as that the participants had more prior knowledge about the LM than the TM. This is in line with our expectations about the impact of the available prior knowledge that exclusively builds on the representation via the LM. However, in the posttest, the scores from the TM exceeded the scores from the LM. Based on the Working Memory Model, we can assume that the TM has played a more facilitating role when supporting the processing of new information and solving of temporal relation in working memory due to the better support of the visuo-spatial sketchpad. Consistently, we can assume that the TM has induced to a lesser extent extraneous cognitive load as compared to the LM. An additional catalyst can be found in the additional video clips that were presented after the pretest, which gave explicit feedback about the problems being solved. This could have fostered in particular the processing and storage of knowledge about the TM.

The scores obtained in the posttests show that the TM is generally a more effective graphical representation of time intervals with respect to these questions. Moreover, the participants spent less time on answering the majority of the questions when time intervals were represented in the TM than in the LM. This reflects a higher efficiency of the TM for visual observation of intervals, as compared with the LM. Furthermore, the efficiency of the TM was less influenced by an increase in the number of intervals, as the correlation between the number of intervals and time consumption was significant in only 6 of the 37 questions. In contrast, this correlation was significant in the majority of questions where time intervals were presented in the LM.

The LM showed advantages in questions that asked about the start point or the end point of intervals. This can be explained as that these properties can be directly observed from the two boundary points of the linear segments. However, the LM was not easy to use in the questions asking about duration and midpoint, which were expressed by the extent and location of the linear segments. It is hard to visually measure and compare the lengths of intervals distributed at different locations. Also, it is not straightforward to compare the midpoints of intervals of different length. The visual identification of these two properties has to take account of both the start point and end point of the linear segments, which may invoke extra visual processing and as such extraneous cognitive load as suggested above. The same applies in the questions about the temporal relations. The identification of the temporal relations in the LM always requires the visual comparison of the two boundary points 
between the linear segments. Therefore, the search for the intervals in a certain temporal relation to a referenced interval is difficult and error-prone. For example, one has to compare a reference interval with other interval scattered in the diagram in order to find the ones that overlap it. This task becomes even more complex when the questions involve two temporal relations. Although the sorting tool in the website might have helped the search process to some extent, finding the correct intervals from the sorted linear segments is still not a straightforward task. Moreover, with increasing number of intervals, such visual observation or comparison has to be done increasing times, which makes the questions with a large number of intervals rather time-consuming. This has been reflected in the positive correlation between the number of intervals and the time that the participant spent on the question, which has been detected in most questions asked in the LM.

In contrast, every interval has a fixed location in the TM. The intervals having a specific property or satisfying a specific temporal relation are located within a specific zone. Therefore, in the questions, the search for the correct intervals is transferred to the identification of specific zones. The question scores indicate that the participants could correctly identify most of the relational zones in the TM. Once the zone has been located, the selection of the intervals within the zone is rather simple and straightforward, which is, moreover, not affected much by the increasing number of intervals presented in the model. This is probably the reason that the correlation between the number of intervals and the time consumption has been detected in fewer questions built on the TM. This finding reveals the potential of the TM in handling a large amount of intervals, which might be difficult for the LM.

The positive learning results of the TM were generated by a group of undergraduate-level participants without prior knowledge about the TM, and after a 20-minute training process. The increase in the knowledge about the TM is reflected in the differences in scores between pretest and posttest. In spite of the biased understanding about the met-by and overlapped-by relations in the TM being detected in the posttest, the participants have been quickly trained to understand and manipulate the TM at a satisfactory level. This provides empirical evidence that the TM and its implementation can be easily comprehended and used by non-expert users. Moreover, the participants overwhelmingly preferred the usage of the TM, which shows that the above-mentioned merits of the TM are perceived and appreciated by the participants.

\section{CONCLUSION AND DIRECTION FOR FUTURE WORK}


This paper elaborated an empirical study of an alternative representation of time intervals, i.e. the TM. The study used a set of questions about time intervals to evaluate the understandability and usability of the TM, as compared with the traditional LM. The results showed that the participants scored better and spent less time in questions building on the TM. Thus, we contend that the TM is more effective and efficient than the LM in terms of interval visualization. Moreover, the TM showed the potential of representing a large amount of time intervals, which seemed to cause extra difficulties when being dealt with in the LM. We believe that these advantages of the TM stem from efficient use of the space, i.e., the properties and relations of intervals are fully expressed by spatial location of points. Intervals within a property range or in a certain relation to other intervals are distributed in a specific zone, which facilitates the visual query process. However, in the LM, information of intervals is represented by not only location but also extent of linear segments. For most questions in this study, one needs to inspect the presented intervals one by one to find out the correct ones. This results in low correctness and efficiency in the question-answering based on the LM. Since these positive results of the TM were obtained by non-expert participants, after a 20minutes training process, we believe that the TM can be easily understood and used by nonexpert users. While this empirical study is based on the comparison between the TM and the LM, its aim is not proposing an entire substitution of the LM with the TM in all application areas. The LM remains an intuitive and founding representation of linear time, and thus is still advantageous in representing simple linear processes and a small number of time intervals. The result from this study, together with findings from our previous research, provides strong support to consider the adoption of the TM in tasks of interval visualization and analysis. It also supports our plans of developing tools based on the TM and releasing them to a broader research and practical contexts.

In future work, further evaluations of the TM will be conducted in different age groups and education levels in order to determine the appropriate user group it can be applied. It would be particularly interesting to evaluate the TM within younger groups, in which the linear time concept is probably less predominant. In addition to the parameters measured in this study, new technologies, such as eye-tracking, can be applied to obtain extra information in research process. This technology can tell more details on the question-answering process. For example, the trajectory of eye-movement can tell the participants' strategies of solving questions. Also, we would be able to discover the areas of the diagram where the participants spend more time to work at. As previously mentioned, there exist other approaches of 
projecting interval to a 2D space, which could be even advantageous than the TM in certain aspects. We do plan to exam them in order to find the optimal ones in different types of tasks. Moreover, we plan to study more applications of the TM and further evaluate it in solving domain-specific problems. One the one hand, the implementation of the TM will be provided to the users that need to analyse massive interval-based data in the field of, for example, computer science, archaeology and geographical information science. Different visualization variables and metaphors can be applied in the TM to represent contextual information. In addition to interval visualization, this implementation will also allow users to formulate temporal queries by creating 2D zones on top of the interval visualization (Qiang, et al., $2012 \mathrm{~b}$ ). On the other hand, attempts will be made to apply the TM in education of history and chronological knowledge. The initial idea is to represent the interval-based knowledge in history textbooks via an interactive website implementing the TM. For example, when the user clicks on a certain chapter or a certain category of events, the website will automatically display the intervals of events in the selected chapters or categories in an interactive TM diagram. It is also interesting that, users can select intervals in the TM using the graphic query devices, to study and analyse the knowledge linked to these intervals. The special merits of the TM combined with interactive visualization techniques can potentially benefit the perception and memorization of the temporal structure of interval-based knowledge.

\section{Acknowledgement}

The authors would like to thank the Research Foundation- Flanders, Belgium (FWO) for supporting this research. They also would like to thank Mr. Wouter Devinck for his excellent work in developing the computer-based test.

\section{References}

Allen, J. F. (1983). Maintaining Knowledge about Temporal Intervals. Communications of the ACM, 26 (11), pp. 832-843.

Baddeley, A., \& Hitch, G. J. (1974). Working memory. In G. Bower (Ed.), Recent Advances in Learning and Motivation. New York: Academic Press.

Bhatt, M., Guesgen, H., Wolfl, S., \& Hazarika, S. (2011). Qualitative Spatial and Temporal Reasoning: Emerging Applications, Trends, and Directions. Spatial Cognition and Computation, 11 (1), pp. 1-14.

Bittner, T. (2002). Approximate Qualitative Temporal Reasoning. Annals of Mathematics and Artificial Intelligence, 36 (1), pp. 39-80.

Cohn, A. G., Bennett, B., Gooday, J., \& Gotts, N. M. (1997). Qualitative Spatial Representation and Reasoning with the Region Connection Calculus. GeoInformatica, 1 (3), pp. 275-316.

De Tré, G., Van de Weghe, N., De Caluwe, R., \& De Maeyer, P. (2006). Towards a Flexible Visualization Tool for Dealing with Temporal Data. In H. Larsen, G. Pasi, D. Ortiz- 
Arroyo, T. Andreasen \& H. Christiansen (Eds.), Flexible Query Answering Systems (Vol. 4027, pp. 109-120). Heidelberg: Springer Berlin.

Freksa, C. (1992). Temporal Reasoning Based on Semi-Intervals. Artificial Intelligence, 54 (1-2), pp. 199-227.

Galton, A. (1990). A Critical Examination of Allen's Theory of Action and Time. Artificial Intelligence, 42 (2-3), pp. 159-188.

Gottfried, B. (2008). Global Feature Schemes in Spatial and Temporal Reasoning. Spatial Cognition and Computation, 8 (1-2), pp. 27-46.

Hochheiser, H., \& Shneiderman, B. (2004). Dynamic query tools for time series data sets: timebox widgets for interactive exploration. Information Visualization, 3 (1), pp. 1-18.

Knauff, M. (1999). The cognitive adequacy of Allen's interval calculus for qualitative spatial representation and reasoning. Spatial Cognition and Computation, 1 (3), pp. 261-290.

Kulpa, Z. (1997). Diagrammatic Representation for a Space of Intervals. Machine Graphics \& Vision, 6 pp. 5-24.

Kulpa, Z. (1997). Diagrammatic Representation of Interval Space in Proving Theorems about Interval Relations. Reliable Computing, 3 (3), pp. 209-217.

Kulpa, Z. (2006). A Diagrammatic Approach to Investigate Interval Relations. Journal of Visual Languages and Computing, 17 (5), pp. 466-502.

Li, X., \& Kraak, M.-J. (2008). The Time Wave. A New Method of Visual Exploration of Geo-data in Time-space. The Cartographic Journal, 45 (3), pp. 193-200.

Ligozat, G. (1994). Temporal reasoning made simpler. Proceedings of the 7th International Conference on IEA/AIE'94, pp. 123-130, Austin, Texas.

Ligozat, G. (1997). Figures for thought: temporal reasoning with pictures. Paper presented at the Workshop on Spatial and Temporal Reasoning (at AAAI-97).

Mayer, R. E., \& Moreno, R. (2003). Nine Ways to Reduce Cognitive Load in Multimedia Learning. Educational Psychologist, 38 (1), pp. 43-52.

Miyake, A., \& Shah, P. (1999). Models of working memory. Mechanisms of active maintenance and executive control. Cambridge:Cambridge University Press.

Neutens, T., Witlox, F., Van de Weghe, N., \& De Maeyer, P. (2007). Space-time opportunities for multiple agents: a constraint-based approach. International Journal of Geographical Information Science, 21 (10), pp. 1061-1076.

Qiang, Y., Delafontaine, M., Asmussen, K., Stichelbaut, B., De Tré, G., De Maeyer, P., \& Van de Weghe, N. (2010). Modelling Imperfect Time Intervals in a Two-Dimensional Space. Control and Cybernetics, 39 (4), pp. 983-1010.

Qiang, Y., Delafontaine, M., Neutens, T., Stichelbaut, B., De Tré, G., De Maeyer, P., \& Van de Weghe, N. (2012a). Analysing Imperfect Temporal Information in GIS using the Triangular Model. The Cartographic Journal, 11(4), pp. 255-272.

Qiang, Y., Delafontaine, M., Versichele, M., De Maeyer, P., \& Van de Weghe, N. (2012b). Interactive Analysis of Time Interval in a Two-Dimensional Space. Information Visualization, 49(3), pp 265-280.

Qiang, Y, Chavoshi, S.H., Logghe, S., De Maeyer, P., \& Van de Weghe, N.. (2013). Multiscale analysis of linear data in a two-dimensional space. Information Visualization, (In press)

Schockaert, S., De Cock, M., \& Kerre, E. E. (2008). Fuzzifying Allen's Temporal Interval Relations. IEEE Transactions on Fuzzy Systems, 16 (2), pp. 517-533.

Stichelbaut, B., \& Bourgeois, J. (2009). The Aerial Imagery of World War One: A Unique Source for Conflict and Landscape Archaeology. Photogrammetrie - Fernerkundung Geoinformation (3), pp. 235-244.

Sweller, J. (1994). Cognitive load theory, learning difficulty and instructional design. Learning and Instruction, 4 pp. 295-312. 
Sweller, J., \& Chandler, P. (1994). Why some material is difficult to learn. Cognition and Instruction, 12 (3), pp. 185-233.

Van de Weghe, N., Docter, R., De Maeyer, P., Bechtold, B., \& Ryckbosch, K. (2007). The Triangular Model as an Instrument for Visualising and Analysing Residuality. Journal of Archaeological Science, 34 (4), pp. 649-655.

Weaver, C., Fyfe, D., Robinson, A., Holdsworth, D., Peuquet, D., \& MacEachren, A. M. (2006). Visual analysis of historic hotel visitation patterns. Proceedings of IEEE Symposium on Visual Analytics Science and Technology(Vast 2006), pp. 35-42, New York.

Weber, M., Alexa, M., \& Muller, W. (2001). Visualizing time-series on spirals. Proceedings of IEEE Symposium on Information Visualization 2001, pp. 7-13, Washington, DC, U.S.A. 


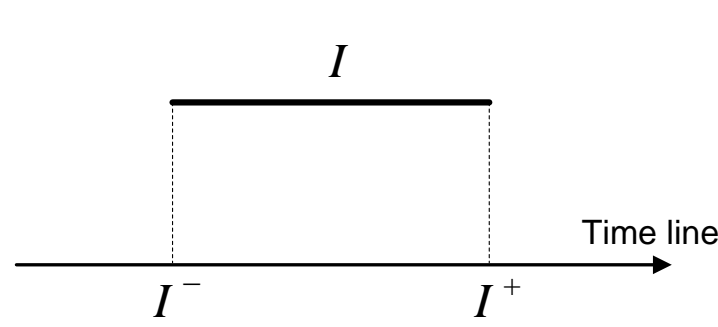

(a)

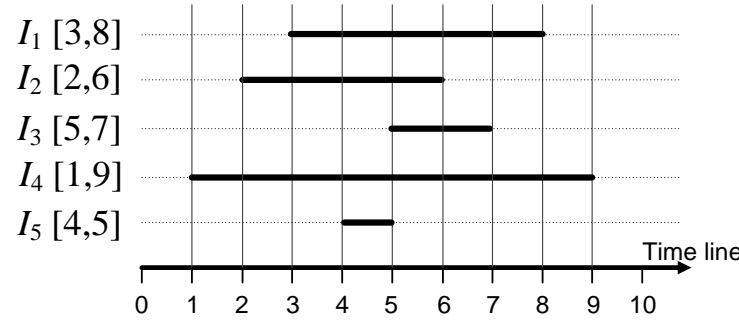

(b)

Figure 1. Representing time interval in the Linear Model. (a) The representation of one interval; (b) the representation of multiple intervals.

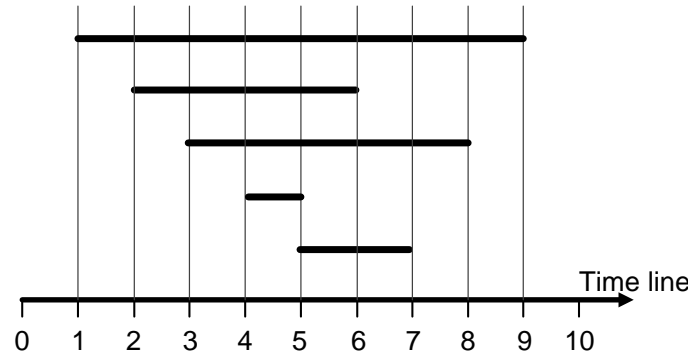

(a)

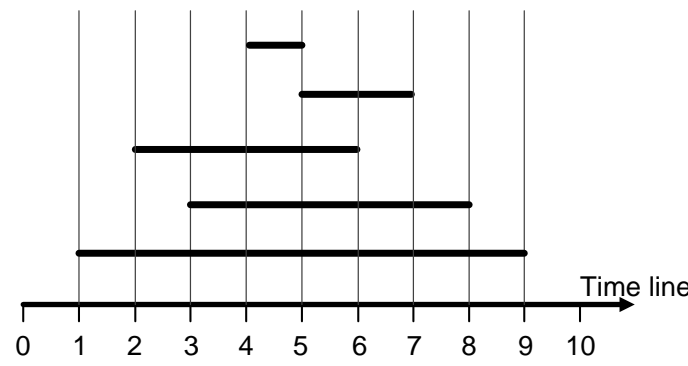

(c)

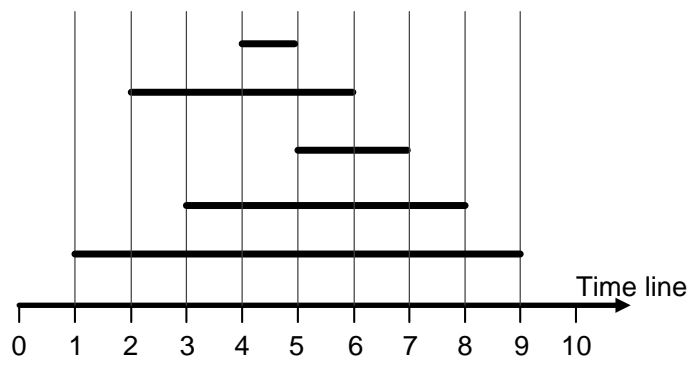

(b)

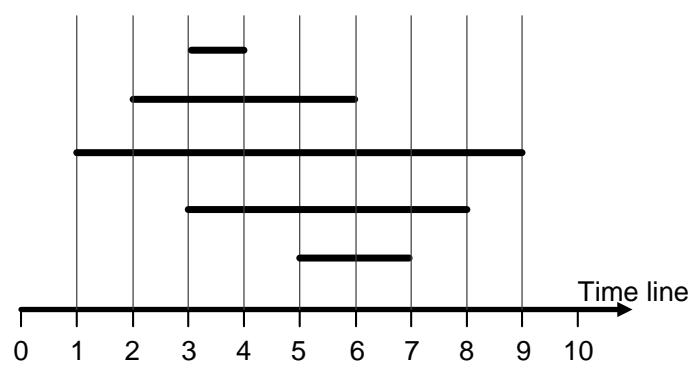

(d)

Figure 2. Four different arrangements of time intervals in the vertical dimension in the Linear Model. (a):ascending start point; (b): ascending end point; (c): ascending duration; (d): ascending midpoint. 


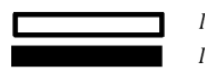

$I_{1}$
$I_{2}$

\begin{tabular}{|c|c|c|}
\hline Representation in the Linear Model & Temporal Relation & Formal Definition \\
\hline & $I_{1}$ before $I_{2}$ & $I_{1}^{+}<I_{2}^{-}$ \\
\hline & $I_{1}$ meets $I_{2}$ & $I_{1}^{+}=I_{2}^{-}$ \\
\hline & $I_{1}$ overlaps $I_{2}$ & $I_{1}^{-}<I_{2}^{-}$and $I_{1}^{+}>I_{2}^{-}$and $I_{1}^{+}<I_{2}^{+}$ \\
\hline & $I_{1}$ starts $I_{2}$ & $I_{1}^{-}=I_{2}^{-}$and $I_{1}^{+}<I_{2}^{+}$ \\
\hline & $I_{1}$ finishes $I_{2}$ & $I_{1}^{-}>I_{2}^{-}$and $I_{1}^{+}=I_{2}^{+}$ \\
\hline & $I_{1}$ during $I_{2}$ & $I_{1}^{-}>I_{2}^{-}$and $I_{1}^{+}<I_{2}^{+}$ \\
\hline & $I_{1}$ equal $I_{2}$ & $I_{1}^{-}=I_{2}^{-}$and $I_{1}^{+}=I_{2}^{+}$ \\
\hline & $I_{1}$ contains $I_{2}$ & $I_{1}^{-}<I_{2}^{-}$and $I_{1}^{+}>I_{2}^{+}$ \\
\hline & $I_{1}$ finished - by $I_{2}$ & $I_{1}^{-}<I_{2}^{-}$and $I_{1}^{+}=I_{2}^{+}$ \\
\hline & $I_{1}$ started - by $I_{2}$ & $I_{1}^{-}=I_{2}^{-}$and $I_{1}^{+}>I_{2}^{+}$ \\
\hline & $I_{1}$ overlapped - by $I_{2}$ & $I_{1}^{-}>I_{2}^{-}$and $I_{1}^{-}<I_{2}^{+}$and $I_{1}^{+}>I_{2}^{+}$ \\
\hline & $I_{1}$ met - by $I_{2}$ & $I_{1}^{-}=I_{2}^{+}$ \\
\hline
\end{tabular}

Figure 3. The representations of temporal relations in the Linear Model.
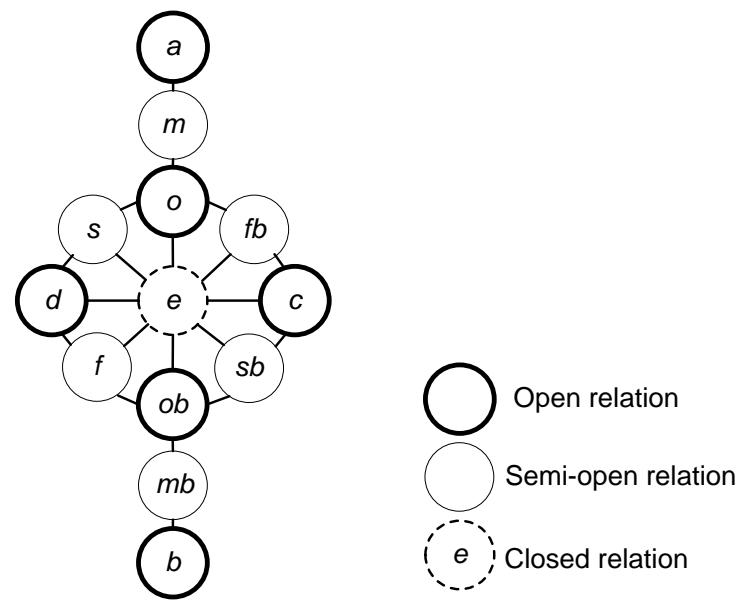

Figure 4: The conceptual neighbourhood structure of thirteen temporal relations.

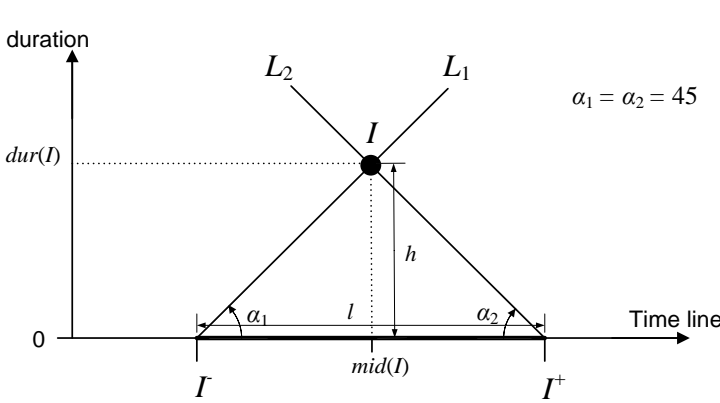

(a)

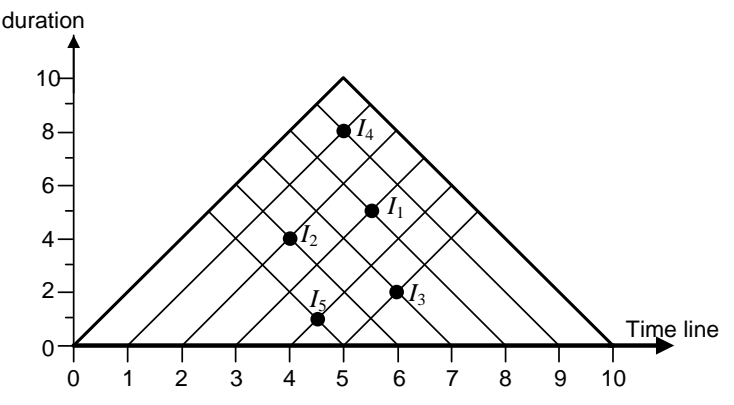

(b)

Figure 5: The representation of intervals in the TM. (a): The construction of an interval point. (b): The representation of the intervals in Figure 1(b) in the TM. 


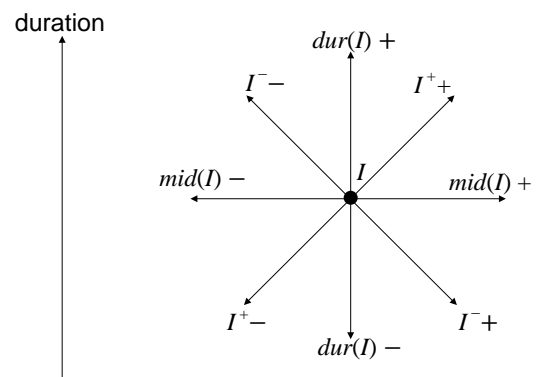

Time line

Figure 6: The meanings of the eight directions in the TM. The minus sign means a decrease, while the plus sign means an increase.

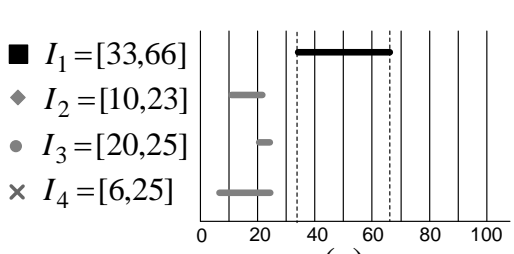

(a)

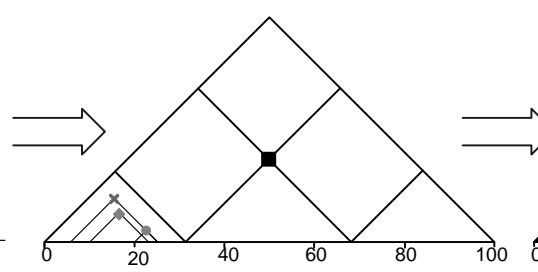

(b)

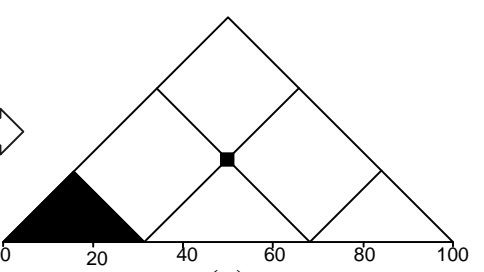

(c)

Figure 7. Temporal relations in the linear model and in TM, taking before as an example.
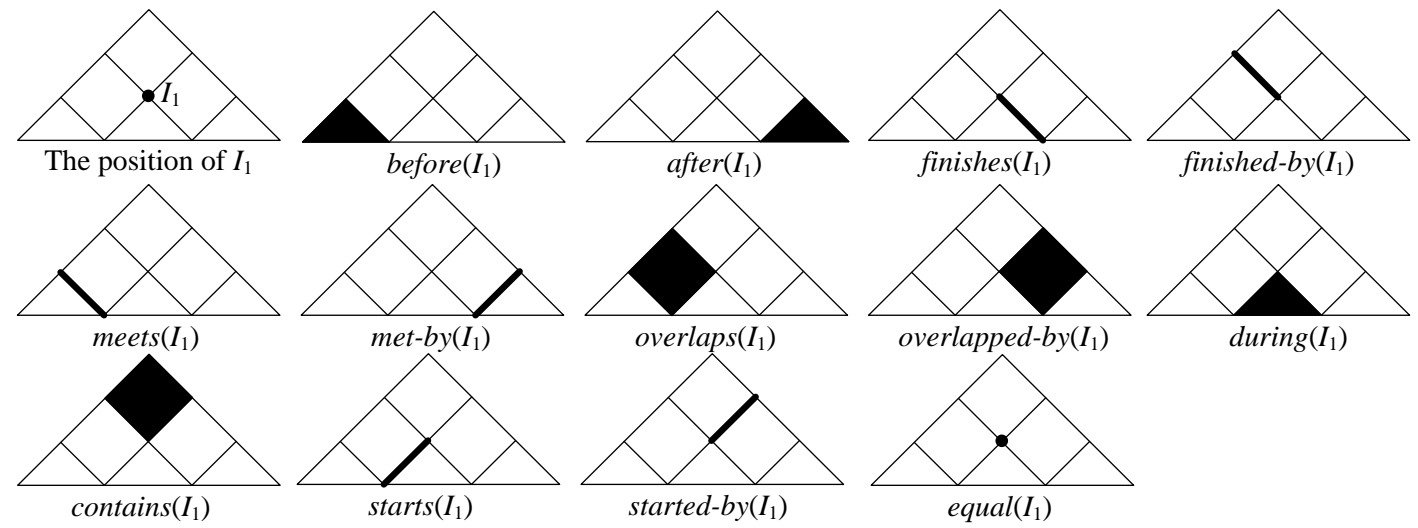

Figure 8: The zones of the temporal relations in the TM.

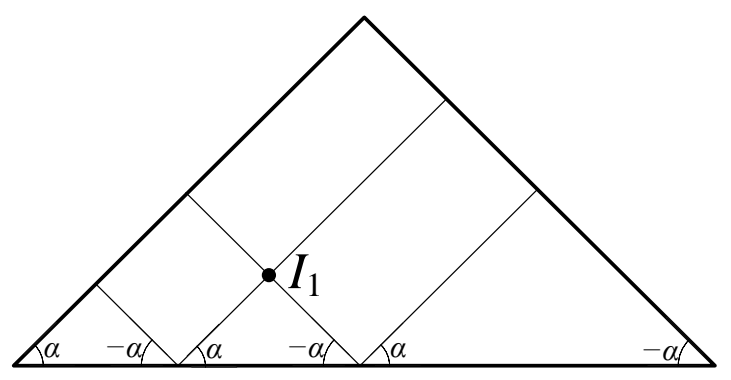

(a)

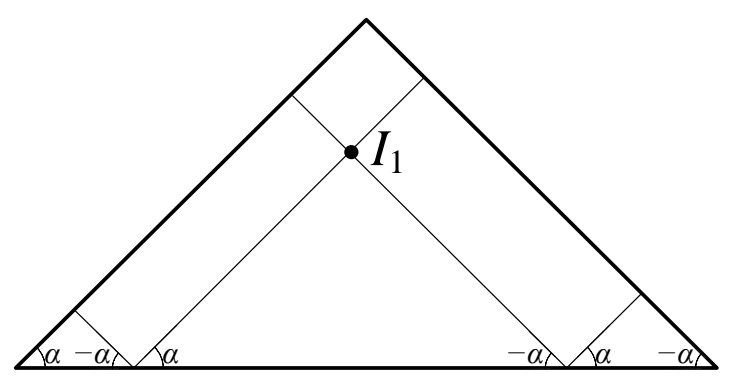

(b)

9: The consistent topological configuration of relational zones in the TM. 


\section{Question 1}

Selecteer de intervallen die starten gedurende [40,60], i.e. $\left\{I \mid 40 \leq I^{-} \leq 60\right\}$.

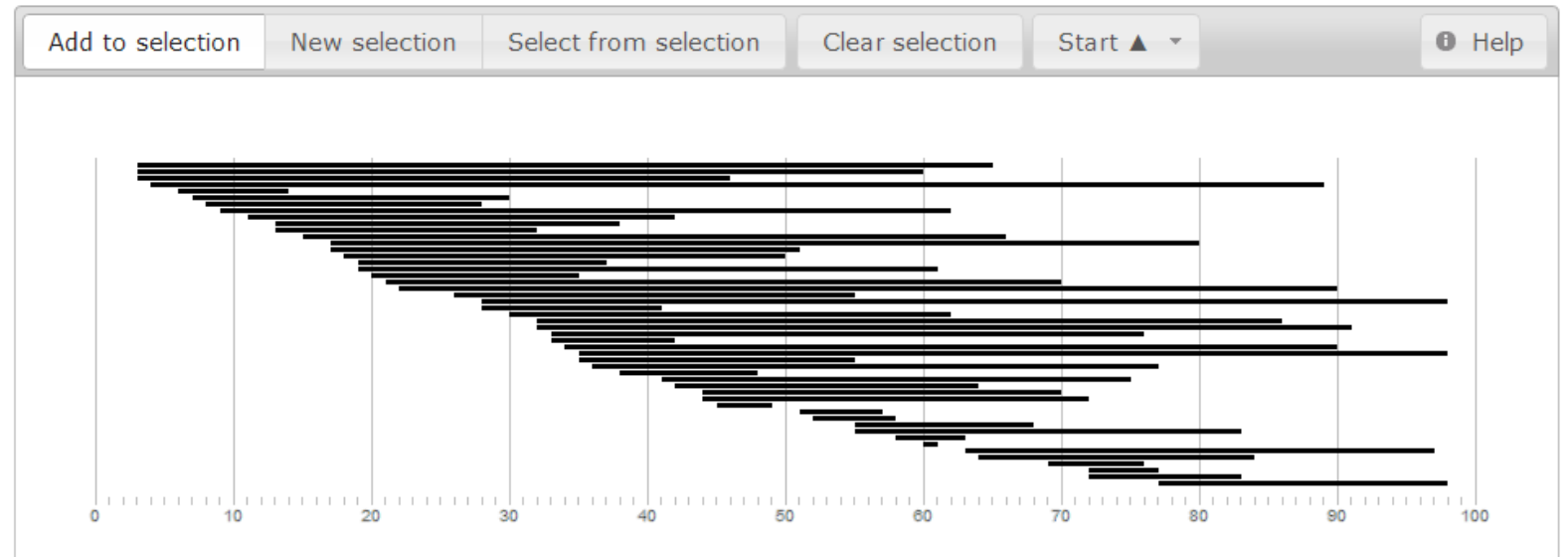

Figure 10: A posttest question on interval property, where intervals are represented in the LM. The translation of the question is 'select the intervals that start during $[40,60]$ '. The time intervals are sorted by ascending start point along the vertical dimension.

\section{Question 2}

Selecteer de intervallen die een duur hebben tussen 40 en 60 , i.e. $\{I \mid 40 \leq \operatorname{dur}(I) \leq 60\}$.

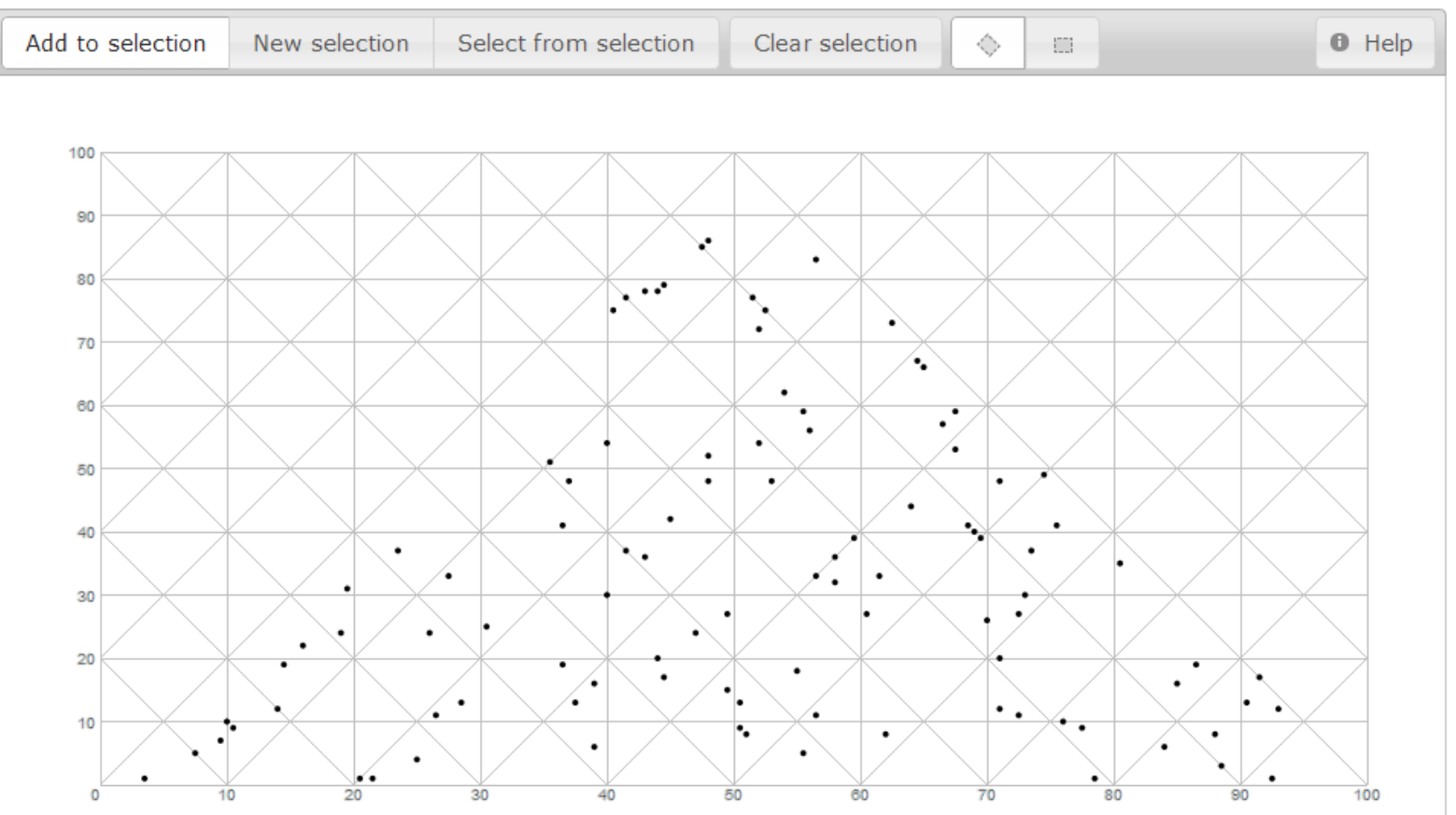

Figure 11: A posttest question on interval property, where intervals are represented in the TM. The translation of the question is 'select the intervals that has a duration between 40 and $60^{\prime}$. 


\section{Question 9}

Selecteer de intervallen die $I_{1}$ overlappen, i.e. $\left\{I \mid I\right.$ overlapt $\left.I_{1}\right\}$.

$I_{1}=[50,80]$

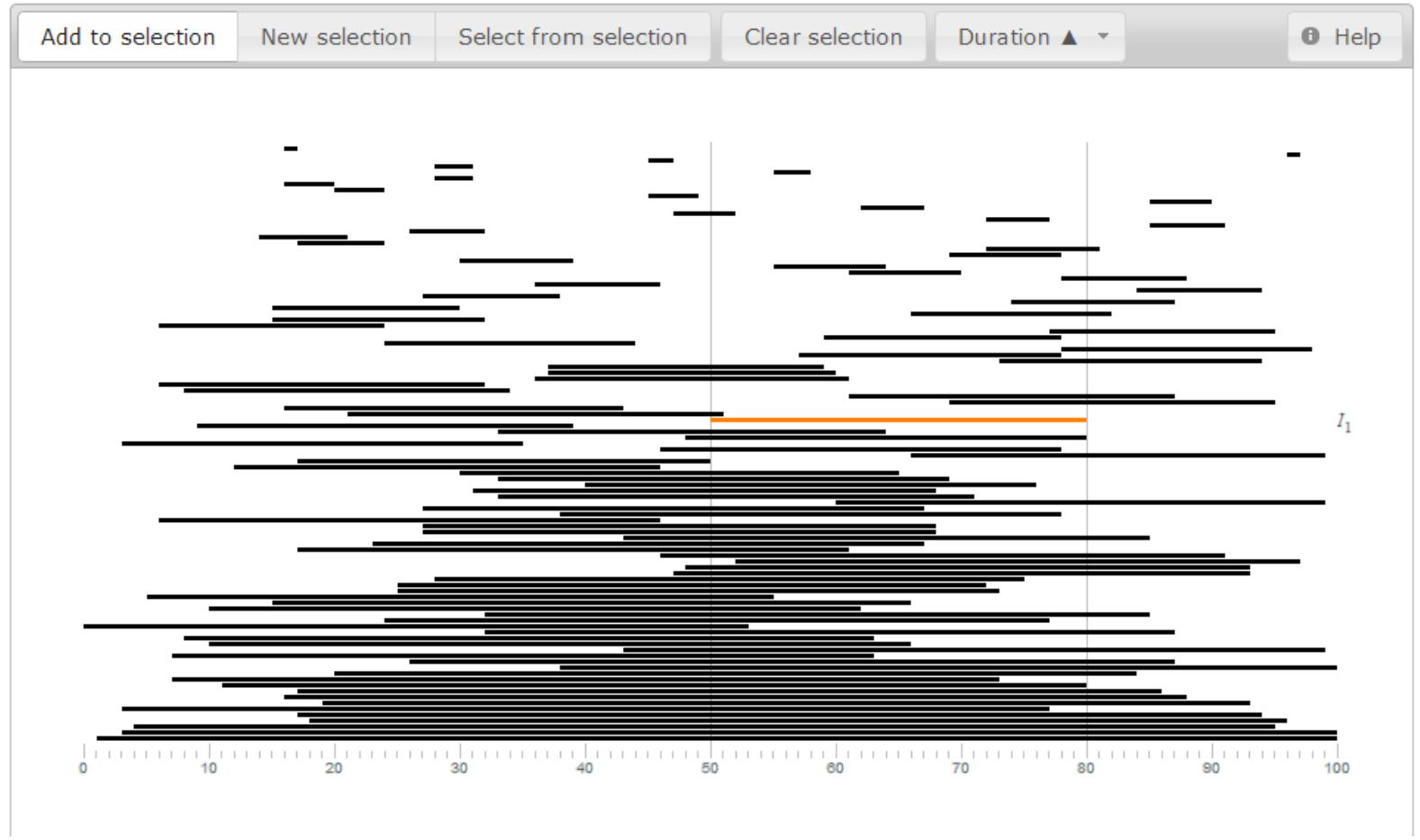

Figure 12: A post-test question on temporal relation, where intervals are represented in the LM. The translation of the question is 'select the intervals that overlap $I_{1}$. The time intervals are sorted by ascending duration along the vertical dimension. 


\section{Question 11}

Selecteer de intervallen gedurende $I_{1}$, i.e. $\left\{I \mid\right.$ I gedurende $\left.I_{1}\right\}$.

$I_{1}=[20,60]$

\begin{tabular}{|l|l|l|l|l|}
\hline Add to selection & New selection & Select from selection & Clear selection & (i) Help
\end{tabular}

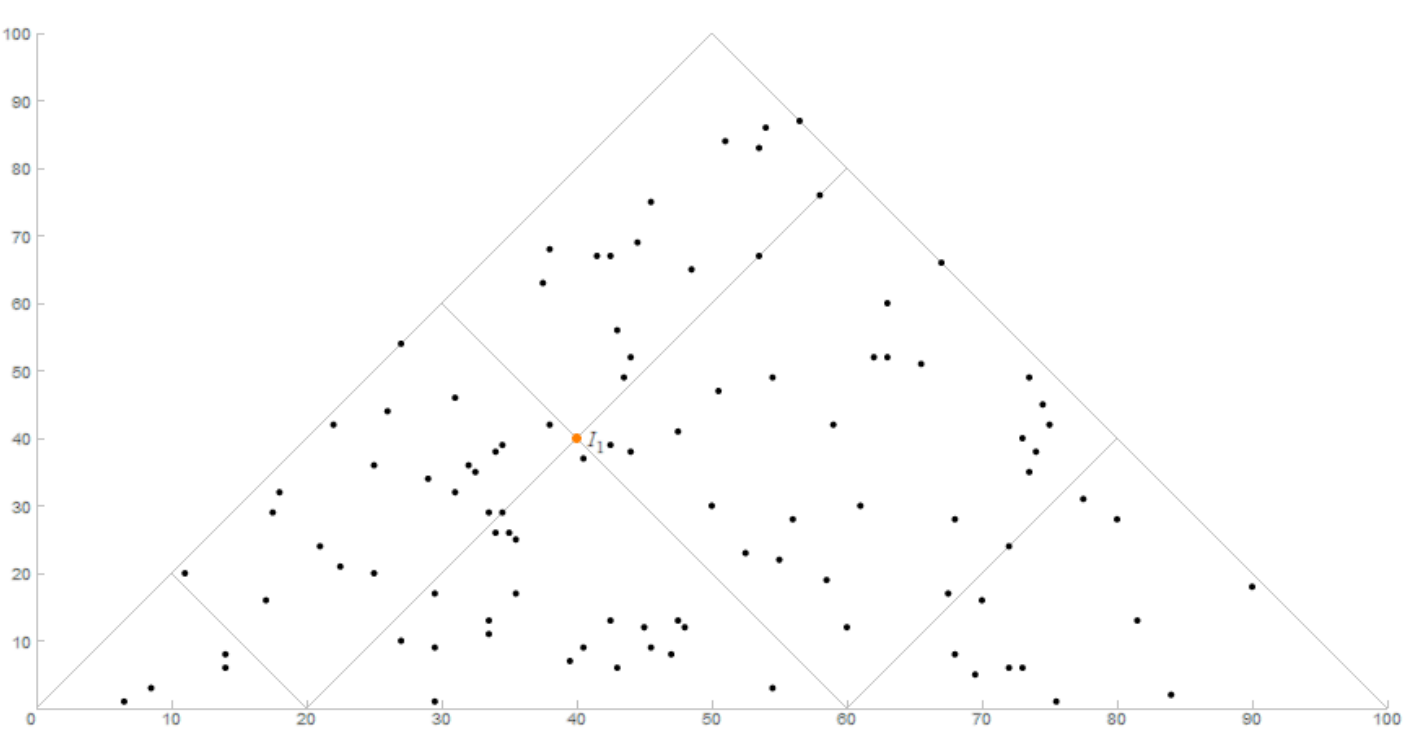

Figure 13: A post-test question on temporal relation, where intervals are represented in the TM. The translation of the question is 'Select the intervals during $I_{1}$ '.

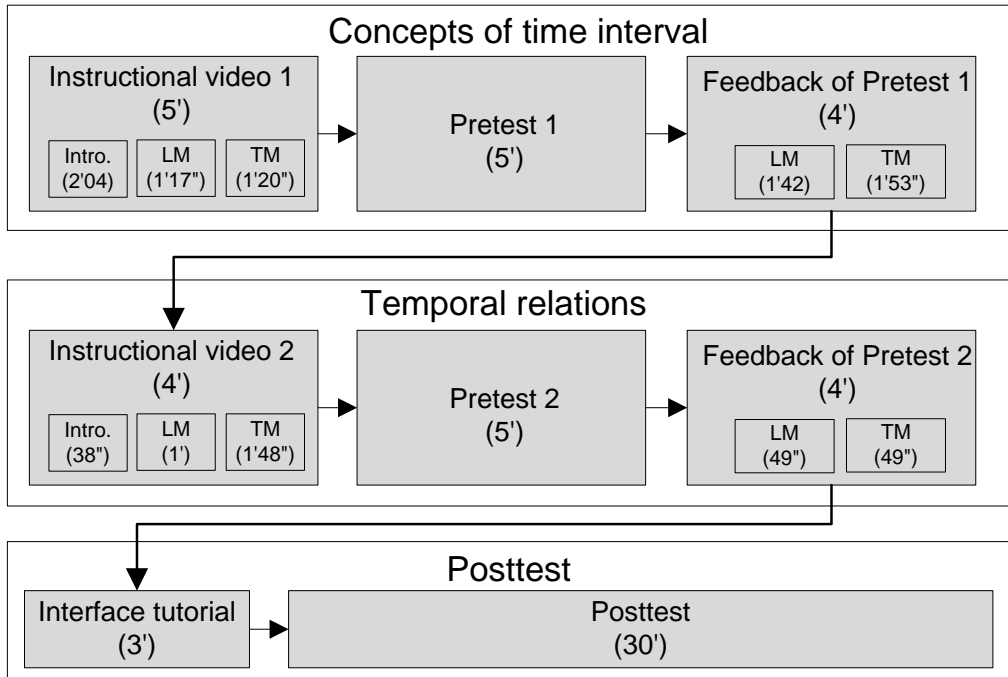

Figure 14: The flowchart of the research procedure. ' represents minute. " represents second. 


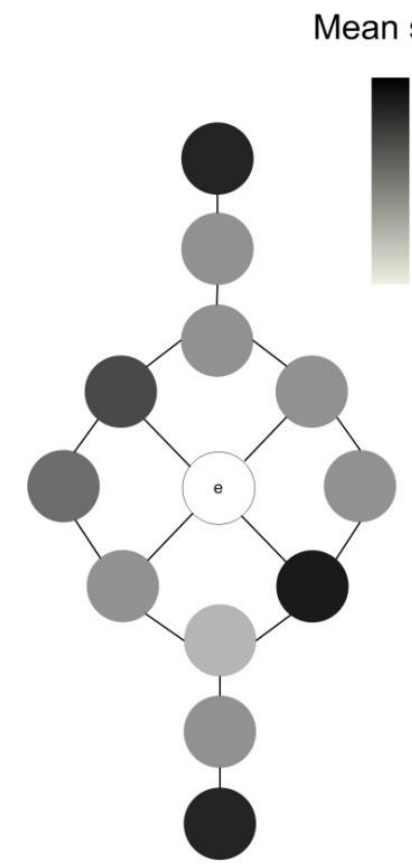

Mean score in LM

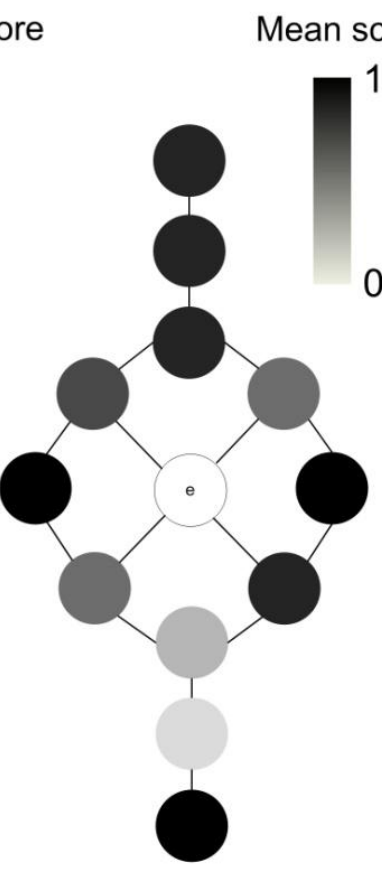

Mean score in TM

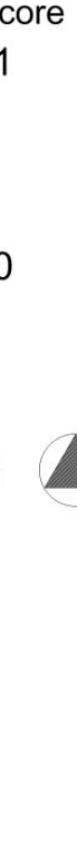

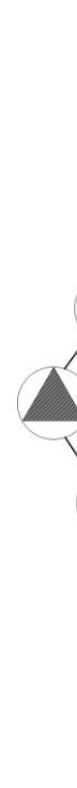

Comparison of
the mean scores

Figure 15: The mean scores of the questions about temporal relations represented as grey scales in the conceptual neighbourhood structure of the temporal relations.

Table 1: The examples of questions in the test

\begin{tabular}{|l|l|l|}
\hline $\begin{array}{l}\text { Type Item number } \\
\text { of question }\end{array}$ & \multicolumn{1}{|c|}{ One item } & \multicolumn{1}{c|}{ Two items } \\
\hline $\begin{array}{l}\text { Interval } \\
\text { property }\end{array}$ & $\begin{array}{l}\text { Please find and select the intervals } \\
\text { that have a start point between } 20 \\
\text { and } 40 .\end{array}$ & $\begin{array}{l}\text { Please find and select the intervals that } \\
\text { have a start point between } 20 \text { and } 40 \text { and } \\
\text { an end point between } 60 \text { and } 80 .\end{array}$ \\
\hline $\begin{array}{l}\text { Temporal } \\
\text { relation }\end{array}$ & $\begin{array}{l}\text { Please find and select the intervals } \\
\text { before the reference interval } I_{1} .\end{array}$ & $\begin{array}{l}\text { Please find and select the intervals that } \\
\text { overlap } I_{1} \text { and are during } I_{2} .\end{array}$ \\
\hline
\end{tabular}


Table 2: Comparing the pretest and the posttest

\begin{tabular}{|l|l|l|}
\hline & \multicolumn{1}{|c|}{ Pretest } & \multicolumn{1}{c|}{ Posttest } \\
\hline Media & Paper-based & Computer-based \\
\hline Function & Training and test & Test \\
\hline Number of questions & 8 & 37 \\
\hline Numbers of intervals & Less (8 to 10) & More (50 to 200) \\
\hline $\begin{array}{l}\text { Linear segment in the Linear } \\
\text { Model }\end{array}$ & Randomly sorted & Can be sorted by the participant \\
\hline Preference of the model & Not asked & Asked \\
\hline Tested parameters & 1 Score of the answers & $\begin{array}{l}\text { 1. Score of the answers } \\
\text { 2. Time used } \\
\text { 3. Preference of the model }\end{array}$ \\
\hline
\end{tabular}


Table 3: The average scores, time consumption and preference in the pretest and posttest

\begin{tabular}{|lllllc|}
\hline Test & \multicolumn{2}{c}{ Mean score } & \multicolumn{2}{c|}{ Mean time } & Preference \\
& LM & TM & LM & TM & \\
\hline Pretest & $0,79 * *$ & 0,73 & N/A & N/A & N/A \\
\hline Posttest & 0,61 & $0,69 * *$ & $123 * *$ & 63 & TM** \\
\hline
\end{tabular}

**The underlying distribution is higher at the significance level of 0.01 (2-tailed) N/A: The parameter is not available in this test 
Table 4: The scores, the time consumption and preference in the posttest, according to specific questions.

\begin{tabular}{|c|c|c|c|c|c|c|c|}
\hline \multirow[t]{2}{*}{ Type } & \multirow[t]{2}{*}{ No. } & \multirow[t]{2}{*}{ Asked concept } & \multicolumn{2}{|c|}{ Mean score } & \multicolumn{2}{|c|}{$\begin{array}{l}\text { Mean time } \\
\text { (seconds) }\end{array}$} & \multirow[t]{2}{*}{ Preference } \\
\hline & & & LM & $\mathrm{TM}$ & LM & TM & \\
\hline \multirow{10}{*}{ 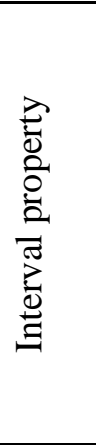 } & 1 & Start point & $0.85 * *$ & 0.70 & 95 & 77 & ---- \\
\hline & 2 & End point & $0.85^{*}$ & 0.71 & 117 & 87 & ---- \\
\hline & 3 & Duration & 0.46 & $0.70 * *$ & $170 * *$ & 91 & $\mathrm{TM}^{* *}$ \\
\hline & 4 & Midpoint & 0.60 & $0.76^{* *}$ & $206 * *$ & 71 & $\mathrm{TM}^{* *}$ \\
\hline & 5 & Start point \& end point & 0.75 & 0.76 & $131 * *$ & 88 & $\begin{array}{ll}--- \\
\end{array}$ \\
\hline & 6 & Start point \& duration & 0.60 & 0.57 & $166^{* * *}$ & 100 & $\mathrm{TM}^{* *}$ \\
\hline & 7 & Start point \& midpoint & 0.73 & 0.73 & $162 * *$ & 80 & $\mathrm{TM}^{* *}$ \\
\hline & 8 & End point \& duration & 0.61 & 0.51 & $177 * *$ & 93 & $\mathrm{TM}^{* *}$ \\
\hline & 9 & End point \& midpoint & 0.76 & 0.69 & $155^{* *}$ & 87 & $\mathrm{TM}^{* *}$ \\
\hline & 10 & Duration \& midpoint & 0.45 & $0.72 * *$ & $169 * *$ & 57 & $\mathrm{TM}^{* *}$ \\
\hline \multirow{27}{*}{ 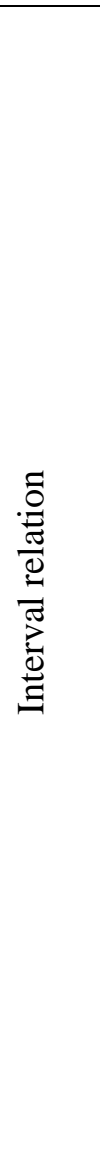 } & 11 & Before & 0.81 & $0.89 * *$ & $80 * *$ & 29 & $\mathrm{TM}^{* *}$ \\
\hline & 12 & Meets & 0.55 & $0.81 * *$ & $83^{* *}$ & 43 & $\mathrm{TM}^{* *}$ \\
\hline & 13 & Overlaps & 0.52 & $0.84 * *$ & $109 * *$ & 36 & TM** \\
\hline & 14 & Finishes & 0.53 & 0.69 & $93 * *$ & 34 & ---- \\
\hline & 15 & Starts & 0.73 & 0.71 & $56^{* *}$ & 38 & ---- \\
\hline & 16 & During & 0.65 & $0.95^{* *}$ & $107 * *$ & 29 & $\mathrm{TM}^{* *}$ \\
\hline & 17 & Contains & 0.58 & $0.90 * *$ & $89 * *$ & 35 & $\mathrm{TM}^{* *}$ \\
\hline & 18 & Started-by & 0.83 & 0.80 & $53 * *$ & 29 & $\mathrm{TM}^{* *}$ \\
\hline & 19 & Finished-by & 0.53 & $0.66^{*}$ & $39 * *$ & 70 & $\mathrm{TM}^{*}$ \\
\hline & 20 & Overlapped-by & 0.48 & 0.42 & $105 * *$ & 36 & $\mathrm{TM}^{* *}$ \\
\hline & 21 & Met-by & $0.57 * *$ & 0.31 & $77 * *$ & 44 & $\mathrm{TM}^{* *}$ \\
\hline & 22 & After & 0.89 & $0.94 *$ & $75^{* *}$ & 45 & $\mathrm{TM}^{*}$ \\
\hline & 23 & Before \& overlap & 0.50 & $0.79 * *$ & $151 * *$ & 72 & $\mathrm{TM}^{* *}$ \\
\hline & 24 & During \& before & 0.62 & 0.67 & $114 * *$ & 51 & $\mathrm{TM}^{* *}$ \\
\hline & 25 & Before \& contain & 0.44 & $0.70 * *$ & $110 * *$ & 49 & $\mathrm{TM}^{* *}$ \\
\hline & 26 & Overlapped-by \& before & 0.39 & 0.36 & $67 * *$ & 123 & ---- \\
\hline & 27 & Before \& after & 0.62 & $0.73 * *$ & $66^{* *}$ & 38 & $\mathrm{TM}^{* *}$ \\
\hline & 28 & Overlap \& during & 0.49 & $0.79 * *$ & $106 * *$ & 52 & $\mathrm{TM}^{* *}$ \\
\hline & 29 & Contain \& overlap & 0.44 & 0.60 & $91 * *$ & 48 & $\mathrm{TM}^{* *}$ \\
\hline & 30 & After \& overlap & 0.57 & 0.44 & $141 * *$ & 80 & $\mathrm{TM}^{* *}$ \\
\hline & 31 & During \& contain & 0.40 & $0.78 * *$ & $89 * *$ & 35 & $\mathrm{TM}^{* *}$ \\
\hline & 32 & During \& overlapped-by & 0.47 & $0.65 * *$ & $86^{* *}$ & 48 & $\mathrm{TM}^{* *}$ \\
\hline & 33 & During \& after & 0.61 & 0.69 & $93 * *$ & 48 & $\mathrm{TM}^{* *}$ \\
\hline & 34 & Contain \& overlapped-by & 0.43 & 0.52 & $94 * *$ & 50 & $\mathrm{TM}^{* *}$ \\
\hline & 35 & Contain \& after & 0.52 & 0.61 & $119 * *$ & 66 & $\mathrm{TM} * *$ \\
\hline & 36 & Overlaps \& overlapped-by & 0.45 & $0.70 * *$ & $135 * *$ & 81 & ---- \\
\hline & 37 & Overlapped-by \& after & 0.41 & 0.39 & $120 * *$ & 89 & $\mathrm{TM}^{* *}$ \\
\hline
\end{tabular}

* The underlying distribution is higher at the significance level of 0.05 (2-tailed)

** The underlying distribution is higher at the significance level of 0.01 (2-tailed)

---- No significant preference detected 
Table 5: The correlation coefficients between the time consumption and the number of intervals, according to specific questions.

\begin{tabular}{|c|c|c|c|c|}
\hline \multirow[t]{2}{*}{ Type } & \multirow[t]{2}{*}{ No. } & \multirow[t]{2}{*}{ Asked concept } & \multicolumn{2}{|c|}{ Correlation coefficient } \\
\hline & & & LM & $\mathrm{TM}$ \\
\hline \multirow{10}{*}{ 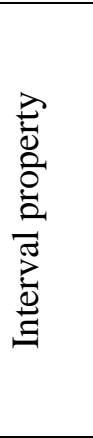 } & 1 & Start point & 0.18 & 0.27 \\
\hline & 2 & End point & 0.15 & -0.12 \\
\hline & 3 & Duration & 0,13 & 0.21 \\
\hline & 4 & Midpoint & $0.34 *$ & 0.22 \\
\hline & 5 & Start point \& end point & $0.30 * *$ & -0.01 \\
\hline & 6 & Start point \& duration & $0.28 *$ & 0.11 \\
\hline & 7 & Start point \& midpoint & $0.47 * *$ & 0.24 \\
\hline & 8 & End point \& duration & 0.08 & 0.01 \\
\hline & 9 & End point \& midpoint & $0.26^{*}$ & -0.08 \\
\hline & 10 & Duration \& midpoint & $0.25^{* *}$ & 0.09 \\
\hline \multirow{27}{*}{ 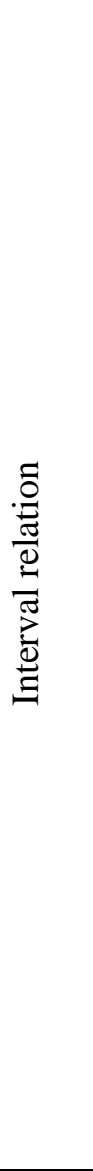 } & 11 & Before & $0.42 *$ & $0.34 * *$ \\
\hline & 12 & Meets & 0.20 & 0.02 \\
\hline & 13 & Overlaps & $0.31 *$ & $0.31 *$ \\
\hline & 14 & Finishes & 0.14 & $0.40^{*}$ \\
\hline & 15 & Starts & $0.47 *$ & 0.26 \\
\hline & 16 & During & $0.26^{*}$ & 0.21 \\
\hline & 17 & Contains & 0.24 & 0.12 \\
\hline & 18 & Started-by & -0.16 & 0.29 \\
\hline & 19 & Finished-by & $0,39 *$ & $-0,31$ \\
\hline & 20 & Overlapped-by & 0.16 & 0.15 \\
\hline & 21 & Met-by & 0.13 & -0.08 \\
\hline & 22 & After & 0.21 & $0.27 *$ \\
\hline & 23 & Before \& overlap & $0.33^{* *}$ & 0.19 \\
\hline & 24 & During \& before & $0.39 * *$ & $0.38 * *$ \\
\hline & 25 & Before $\&$ contain & -0.01 & -0.04 \\
\hline & 26 & Overlapped-by \& before & $0.42 * *$ & 0.18 \\
\hline & 27 & Before \& after & $0.30 * *$ & 0.02 \\
\hline & 28 & Overlap \& during & 0.10 & -0.05 \\
\hline & 29 & Contain \& overlap & 0.20 & 0.14 \\
\hline & 30 & After \& overlap & $0.28^{*}$ & 0.06 \\
\hline & 31 & During \& contain & $0.19 *$ & 0.02 \\
\hline & 32 & During \& overlapped-by & $0.25^{*}$ & 0.04 \\
\hline & 33 & During \& after & -0.05 & -0.01 \\
\hline & 34 & Contain \& Overlapped-by & $0.38 * *$ & $0.28 *$ \\
\hline & 35 & Contain \& after & 0.17 & 0.00 \\
\hline & 36 & Overlaps \& overlapped-by & $0.36^{* *}$ & 0.03 \\
\hline & 37 & Overlapped-by \& after & 0.01 & -0.12 \\
\hline
\end{tabular}

The correlation is at the significance level of 0.05 (2-tailed)

** The correlation is at the significance level of 0.01 (2-tailed) 